International Journal of Engineering \& Technology, $7(2.21)(2018) 361-363$
International Journal of Engineering \& Technology
WPC
Website: www.sciencepubco.com/index.php/IJET
Research paper

\title{
Performance comparison of various denoising filters for brain MRI images
}

\author{
M. Latha ${ }^{1 *}$, S. Arun ${ }^{2}$ \\ ${ }^{I}$ Department of Computer Science \& Engineering, Vels Institute of Science, Technology \& Advanced Studies(VISTAS), Chennai, India. \\ ${ }^{2}$ Department of Computer Science \& Engineering, Vels Institute of Science, Technology \& Advanced Studies(VISTAS), Chennai, India. \\ *Corresponding author E-mail:latha.se@velsuniv.ac.in
}

\begin{abstract}
Communication in the modern age has been done via Visual information which is being transmitted in the form of digital images. The transmitted image often contains noise and need to be preprocessed before applied in algorithms. Image provides some useful structural and functional information about the brain after involving into a simple and non-invasive procedure. Various functional modalities like CT, SPECT and MRI detects some changes in normal metabolism and in flow of blood. If the original image is noisy or has any structural changes, it becomes difficult to identify the required features from the original image and hence preprocessing becomes an essential step. An experimental methodology has been done which compares and classify the various denoising filters.
\end{abstract}

Keywords: CT, SPECT, MRI.

\section{Introduction}

\section{Image and noise}

Digital images have emerged as the key input value in our day to day applications and also in fields of research and technology. Noise modeling gets affected via transmission medias, quantization and radiation [1]. The process of acquiring a data gets varied depending on the environment and its applications. Hence with the large production of images and videos in poor conditions, image enhancement has become an essential step in the process. Additive random noise can be found in images and can be modeled as a gaussian noise. Speckle noise [2] can be found in Ultrasound images and MRI images can have Rician noise [3]. The major focus is on filtering techniques for natural images and its comparisons. An ultrasound image can have speckle noise and it will be correlated as multiplicative noise. Fine details of an image get degraded and contrast resolution will be limited and hence it becomes difficult to detect in images [4]. Linear and NonLinear models are generally used for removing noise form images. Linear models remove noise quickly but edges preservation will not be efficient, Whereas Non linear models can preserve edges in a better way [5].

\section{Performance evaluation criteria}

A filter reduces noise and enhances detail that was blurred [6]. For removing noise in digital images, spatial filtering is found to be an important enhancement technique. It operates on small neighborhood pixels and implemented as convolution masks [7]. Spatial filters use mean filter as a method for denoising, particularly Gaussian noise. Convolution process provides the average values based on the kernel [8]. Neighboring pixel values are calculated and fit into the center pixel location in averaging filter. Various filtering methods were used to remove unwanted noise in images and to estimate the unknown signals. Image denoising has found to be the essential pre-processing step for analyzing an image. The performance of all the filtering techniques and quality of images was evaluated using the following three different quantitative measures.
1. PSNR
2. SNR
3. MSE

\section{Signal and noise ratio}

Peak signal-to-noise ratio (PSNR) defines the ratio between the power of a signal to the power of noise that affects the fidelity of its representation.

$P S N R=20 \log _{10}\left(\frac{M A X_{f}}{\sqrt{M S E}}\right)$

The mean square error will be the bias square value added with variance value. $M S E=\frac{1}{m n} \sum_{0}^{m-1} \sum_{0}^{n-1}\|f(i, j)-g(i, j)\|^{2}$

\section{Classification of denoising filters}

\section{Spatial filtering}

Spatial filters can be used to remove noise from an image and further classified into linear and non-linear filters. Spatial filters can be applied in both static and dynamic images [9].

\section{Non-Linear filters}

Edge values will be preserved and found to be efficient than linear filters. Noise levels get reduced without blurring the images which 
makes the design to be difficult. Without explicit identification noise can be removed in Non-linear filters. Various non-linear filters like Rank selection, Weighted and relaxed Median was also used [10]

\section{Linear filters}

Linear filters blur the edges and other pixel details. Each pixel gets replaced with a linear combination of its neighbor pixels using convolution kernel. Convolution in Linear filtering of a signal can be expressed as.

$$
\mathrm{y}(\mathrm{t})=(h r \cdot x t-r d r)
$$

\section{Wiener}

Wiener filter is the linear filter which is used for images which gets degraded by additive noise [11]. If the signal is found to be smooth, then wiener filtering can be used. Spatial smoothing can be used for wiener filter and wavelet denoising was also used [12]. Fig. 1 shows the original image and filtered image using Wiener Filter.
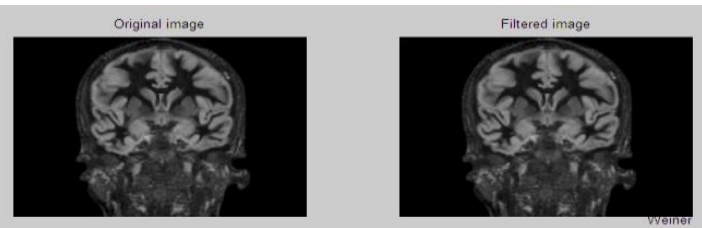

Fig. 1: Output image of wiener filter

\section{Gaussian}

Each pixel will be a sum of true pixel value and a random Gaussian value evenly distributed over the signal. Fig.2 Shows the original image and filtered image using Gaussian Filter.
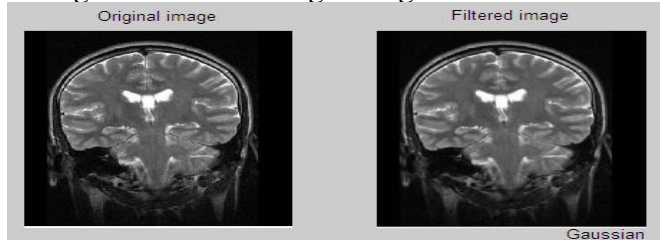

Fig. 2: Output image of gaussian filter

\section{Mean filter (Average filter)}

It is a type of spatial filtering operation which replaces the centre value by the average values of its nearest pixels including itself.
Average filter removes even small variations by performing smoothing on an image. It uses a convolution filter around a kernel representing the shape and size of the neighborhood to be sampled when calculating the mean [13]. Fig.3 Shows the original image and filtered image using Mean Filter.
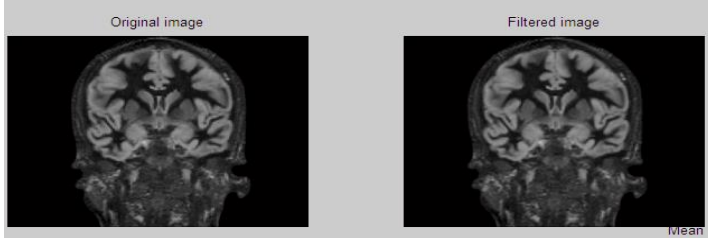

Fig. 3: Output image of mean filter

\section{Median filter}

It is a type of non-linear filter technique used to remove noise. The random valued impulse noise can be removed by median filter where the value of corrupted pixel will be replaced by median value of corresponding window. The median is calculated by pixel sorting and then replaced with the middle pixel value. It is found to be less effective for those images affected with gaussian noise $[13,14]$. Fig.4 Shows the original image and filtered image using Median Filter.
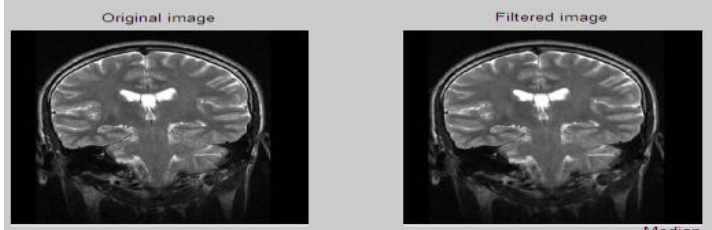

Fig. 4: Output image of median filter

\section{Results and discussion}

Brain MRI images has been taken as the input and various filters has been applied calculating PSNR, SNR and MSE values. Various figures showing the output images obtained for Wiener, Gaussian ,Mean and Median Filters has been shown in Fig 1,2,3,4 respectively. Table 1 shows the performance values (PSNR, SNR, and MSE) obtained by applying Wiener filter. Similarly, performance measures are calculated by applying other filters and its PSNR value comparison is shown in Fig 5. SNR and MSE shows an approximate equal range of values for all filters.

Table 1: Performance Values obtained for Wiener Filter

\begin{tabular}{|c|c|c|c|c|}
\hline Image & & PSNR & SNR & MSE \\
\hline 1 & & 38.17 & 15.88 & 37.89 \\
\hline 2 & & 38.41 & 12.95 & 57.35 \\
\hline 3 & & 31.77 & 14.31 & 48.48 \\
\hline 4 & & 36.52 & 15.89 & 40.91 \\
\hline 5 & & 41.83 & 14.75 & 46.48 \\
\hline 6 & & 32.48 & 13.13 & 56.23 \\
\hline 7 & & 30.43 & 13.53 & 53.65 \\
\hline 8 & & 51.66 & 14.85 & 36.86 \\
\hline 9 & & 51.5 & 13.42 & 40.95 \\
\hline 10 & & 36.91 & 12.81 & 58.34 \\
\hline 11 & & 39.03 & 13.88 & 42.27 \\
\hline 12 & & 30.5 & 11.39 & 68.69 \\
\hline 13 & & 32.82 & 11.9 & 64.73 \\
\hline 14 & & 35.39 & 12.92 & 57.1 \\
\hline 15 & & 31.12 & 10.51 & 75.98 \\
\hline PSNR & - & \multicolumn{3}{|c|}{ Peak Signal to Noise Ratio } \\
\hline SNR & - & \multicolumn{3}{|c|}{ Signal to Noise Ratio } \\
\hline MSE & - & \multicolumn{3}{|c|}{ Mean Square Error } \\
\hline
\end{tabular}




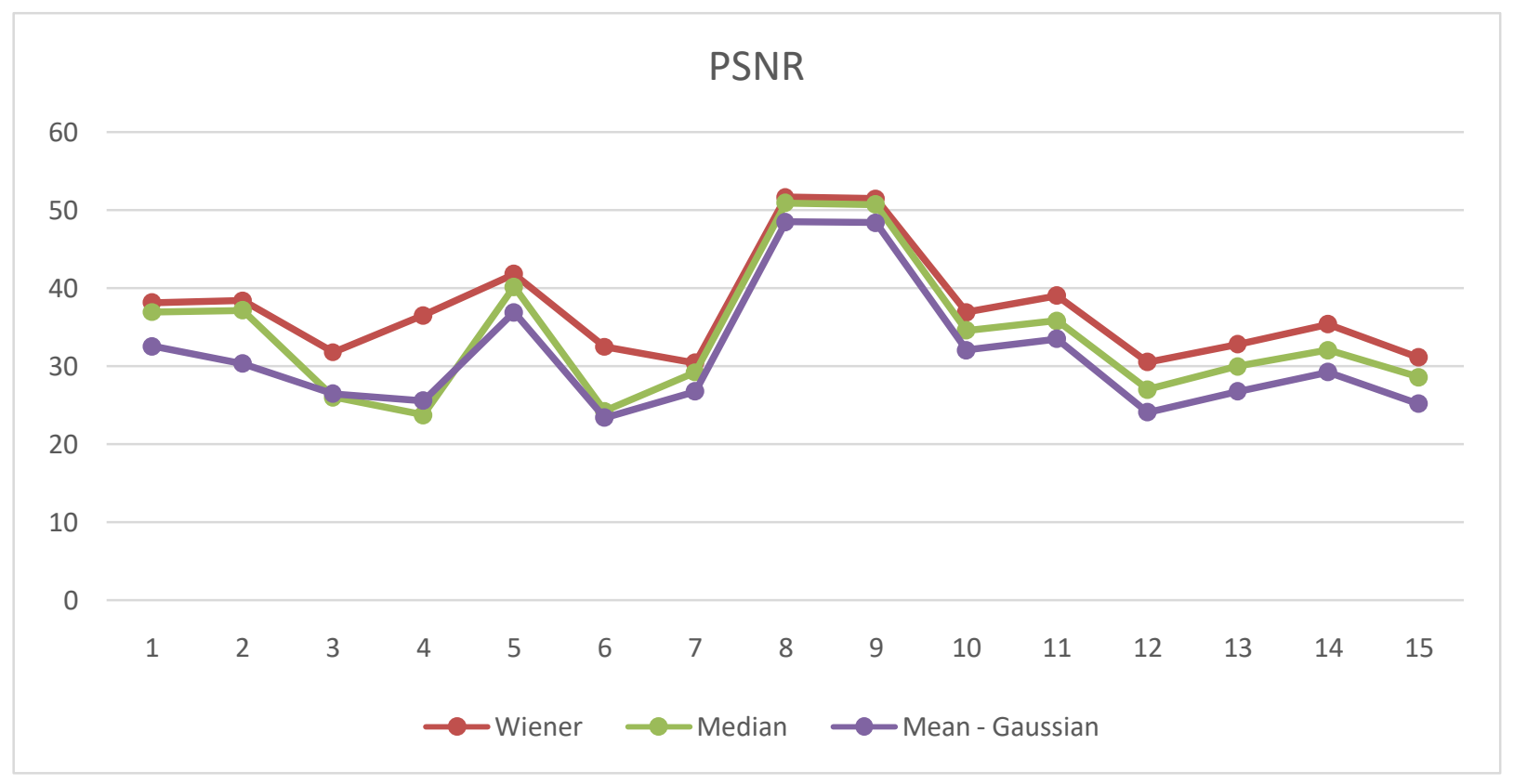

Fig. 5: Graph showing the PSNR values for various filters

\section{Conclusion}

Understanding the finer details in image has found to be the essential step in image processing in all domains. Hence an image should be preprocessed before applied in algorithmic evaluations. Each pixel holds some data and while removing noise from such images, data should not get distracted. Various filters have been applied for Brain MRI Images and the performance was measured using the PSNR, SNR and MSE values. In this paper filters were applied for all input MRI images and values were compared and shown in charts which will help us to use the denoised image for further processing.

\section{References}

[1] Motwani MC, Gadiya MC, Motwani RC \& Harris FC Jr. "Survey of Image Denoising Techniques", Proceedings of GSPX, (2004), pp.27-30.

[2] Guo H, Odegard JE, Lang M, Gopinath RA, Selesnick IW \& Burrus CS, "Wavelet based speckle reduction with application to SAR based ATD/R", First Int'l Conf. on Image Processing, Vol.1, (1994), pp.75-79.

[3] Nowak RD, "Wavelet Based Rician Noise Removal", IEEE Transactions on Image Processing, Vol.8, No.10, (1999).

[4] Yue, Croitoru MM, Bidani A, Zwischenberger JB \& Clark JW, "Nonlinear multiscale wavelet diffusion for speckle suppression and edge enhancement in ultrasound images", IEEE transactions on medical imaging, Vol.25, No.3,(2006), pp.297-311.

[5] Suganthi M \& Deepa P, "Performance Evaluation of Various Denoising Filters for Medical Image", International Journal of Computer Science and Information Technologies, Vol.5, No.3, (2014), pp.4205-4209.

[6] Hwang H \& Haddad R, "Adaptive Median Filters: New Algorithms and Results", IEEE Trans. Image Processing, Vol.4, No.4, (1995), pp.499-502.

[7] Sangwin S \& Horne R, the Color Image Processing Handbook, Chapman \& Hall, (1998).

[8] Donoho DL, "Denoising by soft-thresholding", IEEE Trans. Information Theory, Vol.41, No.3, (1995), pp.613- 627.

[9] Chandel R \& Gupta G, "Image Filtering Algorithms and Techniques: A Review", International Journal of Advanced Research in Computer Science and Software Engineering, Vol.3, No.10, (2013).

[10] Ben Hamza A, Luque P, Martinez J \& Roman R, "Removing noise and preserving details with relaxed median filters", J. Math. Imag. Vision, Vol.11, No.2, (1999), pp.161-177.
[11] Kailath T, Equations of Wiener-Hopf type in filtering theory and related applications, in Norbert Wiener: Collected Work, vol. III, P.Masani, Ed. Cambridge, MA: MIT Press, (1976), pp.63-94.

[12] Strela V, "Denoising via block Wiener filtering in wavelet domain", 3rd European Congress of Mathematics, Barcelona, (2000).

[13] Tania S \& Rowaida R, “A comparative study of various image filtering techniques for removing various noisy pixels in aerial image", International Journal of Signal Processing, Image Processing and Pattern Recognition, Vol.9, No.3, (2016), pp.113124.

[14] Saxena P, Pavel DG, Quintana JC \& Horwitz B, "An automatic threshold-based scaling method for enhancing the usefulness of TcHMPAO SPECT in the diagnosis of Alzheimer's disease", International Conference on Medical Image Computing and Computer-Assisted Intervention, (1998), pp.623-630. 